\title{
Integrating quality and maintenance decisions in a production-inventory model for deteriorating items
}

\author{
HESHAM K. ALFARES*, SYED NAVEED KHURSHEED \\ and SYED MOBASHER NOMAN
}

\begin{abstract}
Systems Engineering Department, King Fahd University of Petroleum and Minerals, PO Box 5067, Dhahran 31261, Saudi Arabia
\end{abstract}

(Revision received September 2004)

\begin{abstract}
In typical production-inventory models of deteriorating items, deterioration of the production process has not been considered. In this paper, a model is proposed in which both the produced items and the production equipment deteriorate. When the production system deteriorates, it shifts to an outof-control state and begins to produce a proportion of defective items, necessitating corrective maintenance action. A model is formulated to integrate several realistic aspects, including item and process deterioration, varying demand and production rates, quality, inspection, and maintenance. A heuristic solution algorithm is developed to determine the production and inspection schedules, and a numerical example is solved.
\end{abstract}

\section{Introduction}

In many inventory models, it is assumed that the items can be stored indefinitely without any risk of deterioration. However, certain types of items undergo changes while in storage so that, with time, they become partially or entirely unfit for use. Deterioration refers to damage, spoilage, vaporization, or obsolescence of the products. There are several types of items that will deteriorate if stored for extended periods of time. Examples of deteriorating items include metal parts, which are prone to corrosion and rusting, and food items, which are subject to spoilage and decay. Electronic components and fashion clothing also fall into this category, because they can become obsolete over time and their demand will typically decrease drastically.

Inventory control for deteriorating items has been a well-studied problem. Numerous optimal and heuristic approaches have been developed for modeling and solving different variations of this problem. Several criteria have been proposed for classifying deteriorating inventory models, including the following:

1. Fixed/random lifetime perishability.

2. Deterministic/probabilistic and constant/varying demand.

3. Constant/varying deterioration rate.

4. Single/multiple items.

*Corresponding author. Email: hesham@ccse.kfupm.edu.sa 
5. Single/multiple time periods.

6. Shortage/no shortage models.

7. Purchase/manufacturing models.

8. Economic models: price discounts, payment delay, and inflation.

9. Queuing models.

Typically, the effects of producing bad quality items or the restoration of the process (i.e., inspection and maintenance) have not been considered in previous studies of inventory deterioration. Therefore, there seems to be a genuine need for looking into this aspect in order to make the analysis closer to reality. Thus, the problem considered in this paper is to incorporate quality, inspection, and maintenance into a deteriorating inventory model with a decreasing demand rate and a constant deterioration rate. First, a model will be formulated, and then a methodology will be presented to determine the optimal parameters for the new model, such as the cycle time and the number of inspections.

A review of the most recent related literature is given in section 2. A mathematical model is developed in section 3. The solution methodology is presented, illustrated, and analysed in section 4. Finally, conclusions and suggestions for future research are given in section 5 .

\section{Literature review}

Goyal and Giri (2001) provided the most recent review of the literature on deteriorating inventory models published since the early 1990s, classifying them on the basis of shelf-life characteristic and demand variations. For many products, the influence of various market trends on demand is a primary factor in deterioration. Goswami and Chaudhuri (1992) formulated and analytically solved the inventory replenishment problem for a deteriorating item with linearly time-varying demand, finite shortage cost, and equal replenishment intervals. Wee (1995) proposed a replenishment policy for a deteriorating product where demand declines exponentially over a fixed time horizon with constant deterioration and complete or partial backordering. Models were numerically solved and the policies compared assuming that shortages are allowed. Su and Lin (2001) optimally solved a production-inventory model for deteriorating products with shortages, in which the demand is exponentially decreasing and the production rate at any time depends on both the demand and the inventory level. They determined optimal expressions for the production period, maximum inventory level, backorder level, and average total cost. Because of demand and production rate variability, the model of $\mathrm{Su}$ and Lin (2001) forms an important component of our model.

The appropriate deterioration distribution is used to model the nature of the deterioration rate. Hariga (1995) proposed formulations and iterative solutions for a generalized inventory lot-sizing problem and considered three deterioration functions: Erlang, uniform, and exponential. Benkherouf and Mohammed (1996) analysed the inventory replenishment problem with shortages, a constant deterioration rate, and an increasing demand. They presented two optimum solution methods: an exact algorithm, and a dynamic programming algorithm. Hwang (1999) formulated and numerically solved a model with a fixed deterioration rate under both the FIFO and LIFO issuing policies. Abad (2000) formulated a problem 
with exponentially deteriorating items, deterministic demand, and partial backordering as a convex nonlinear programming (NLP) model.

Shortages are either allowed or not allowed; and partially or completely backordered; or lost. Padmanabhan and Vrat (1995) developed stock-dependent demand models and determined economic order quantity (EOQ) expressions for deteriorating items with complete, partial, and no backlogging. Wee (1995) and Chu and Chen (2002) allowed shortages for all periods except the final one, assuming a fixed ratio of backorders to lost sales. Chu and Chen (2002) showed that the inventory carrying cost is proportional to the cost of deteriorated items, and proposed a near-optimal closed-form approximate solution. Wang (2002) argued that assuming a fixed fraction of backorders is not reasonable, since the length of the waiting period is the main factor in whether or not customers accept backordering. He introduced a timedependent partial backlogging rate and opportunity cost due to lost sales.

Several previous studies were focused on maintenance and inspection policies for deteriorating production systems. Lee and Rosenblatt (1989) determined the production and inspection schedules for a system in which the cost of maintenance depends on detection delay, i.e., number of periods in the out-of-control state. The optimum maintenance schedule is determined as a function of the cost of defective items, the cost of restoration, and the mean time until the system is out-of-control. The model of Lee and Rosenblatt (1989) will constitute a major element of our model. Posner et al. (1994) analysed a number of producing machines that are either stopped for repair when they fail naturally, or stopped deliberately due to capacity limitations or other considerations. Iravani and Duenyas (2002) formulated a Markov decision process to jointly plan production and maintenance of a single deteriorating machine with an increasing failure rate to satisfy a stochastic demand. In their model, machine deterioration is assumed to decrease its production rate and increase the time and cost of its maintenance.

Quality control was integrated with production/inventory control and maintenance scheduling in several models. Rahim (1994) presented a model to simultaneously determine the production lot size, inspection schedule, and control chart parameters for a deteriorating production process with a general time-to-failure distribution and increasing failure rate. The objective is to minimize the expected total cost of quality control and inventory control per unit time. Ben-Daya (1999) extended this work by assuming that the reduction in the age of a deteriorating system is proportional to the level of preventive maintenance. Wang and Sheu (2001) determined the optimal lot size and inspection threshold for deteriorating production equipment, assuming the first group of items of each batch is not inspected.

Rahim and Ben-Daya (2001) provided the only previous model in which the deterioration of both the items produced and the production equipment is considered. They combined the deteriorating inventory model of Misra (1975) with the deteriorating equipment model of Rahim (1994). The combined model is used to determine the optimal lot size, inspection schedule, and control chart limits, with the assumptions of arbitrary distribution of deterioration times, normal distribution of the quality characteristic, and constant demand and production rates. In comparison, we assume that both the demand rate and the production rate are time dependent, with an exponentially decreasing demand and demand-dependent production rate. In this paper, the effects of equipment deterioration, variable demand and 
production rates, and inspection and maintenance decisions will be included in an integrated model of deteriorating inventory.

\section{Model development}

In order to develop the integrated model, we will combine and extend two existing models. The first is the production-inventory model presented by $\mathrm{Su}$ and Lin (2001) for deteriorating items in which the production rate depends on both the demand rate and the inventory level. The second is the production-inspection model of Lee and Rosenblatt (1989) in which the inspection intervals are equal and the cost of system maintenance is a function of the detection delay. For our model, the following aspects will be incorporated:

1. Considering the possibility of producing bad quality items, given the occurrence of a shift in the process to the out-of-control state.

2. Incorporating both the production-inventory schedule and the inspectionmaintenance schedule, since the process will not return to the in-control state unless it is inspected and restored.

3. Modeling both deteriorating production equipment as well as deteriorating inventory items.

4. Assuming the production rate to be a function of the demand rate, and both to be varying with time.

\subsection{Notation}

$\begin{aligned} \alpha & \text { fraction of bad items produced in the out-of-control state, } 0 \leq \alpha \leq 1 \\ D(t) & \text { demand rate at time } t, t \geq 0 \\ E(N) & \text { expected number of defective items produced per unit time during } \\ & \text { the production interval }\left(0, t_{1}\right) \\ I_{\mathrm{m}} & \text { maximum inventory level } \\ I(t) & \text { inventory level at any time } t \\ T C & \text { total average cost of the system per unit time } \\ k_{\mathrm{d}} & \text { deterioration cost per unit } \\ k_{\mathrm{f}} & \text { cost of each inspection } \\ k_{\mathrm{h}} & \text { inventory holding (carrying) cost per unit per unit time } \\ k_{\mathrm{q}} & \text { cost resulting from producing a defective item } \\ k_{\mathrm{S}} & \text { setup cost for each new cycle } \\ C_{\mathrm{D}} & \text { deterioration cost per unit time } \\ C_{\mathrm{F}} & \text { inspection cost per unit time } \\ C_{\mathrm{H}} & \text { inventory holding cost per unit time } \\ C_{\mathrm{Q}} & \text { quality cost (due to defective items) per unit time } \\ C_{\mathrm{R}} & \text { restoration (maintenance) cost per unit time } \\ C_{\mathrm{S}} & \text { setup cost per unit time } \\ 1 / \mu & \text { mean time until the shift to the out-of-control state } \\ n & \text { total number of inspections per cycle, all performed in the interval } \\ P(t) & \left.\text { production rate at time } t, 0 \leq t \leq t_{1}\right] \\ \tau & \text { detection delay, i.e., the elapsed time from the shift to the out- } \\ & \text { of-control state to the inspection/restoration time }\end{aligned}$




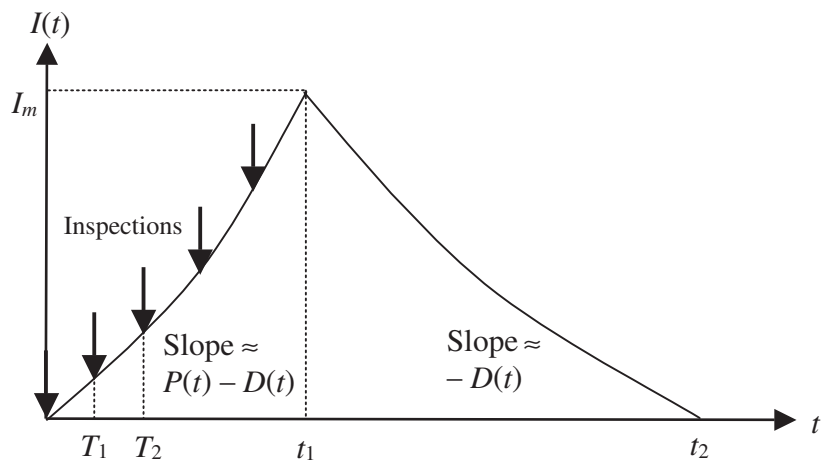

Figure 1. The production-inventory cycle.

$t_{1} \quad$ production time cycle shown in figure 1

$t_{2} \quad$ cycle time

$T_{i} \quad$ time of the $i$ th inspection, $i=0, \ldots, n-1, T_{0}=0, T_{n}=t_{1}$

$\theta \quad$ fraction of inventory items that deteriorate per unit time, $0 \leq \theta \leq 1$

$R(\tau) \quad$ restoration cost as a linear function of detection delay $\tau$

\subsection{Assumptions}

1. A single item is considered, which is subject to a constant $\theta$.

2. $D(t)$ is known and decreases exponentially over time. That is, at time $t, t \geq 0$, $D(t)=A \mathrm{e}^{-\lambda t}$, where $A$ is the initial demand rate and $\lambda$ is the rate of demand decrease, $0 \leq \lambda \leq 1$.

3. $P(t)$ at any instant depends on $D(t)$, i.e., at time $t, t \geq 0, P(t)=a+b D(t)$, $a>0,0 \leq b<1$.

4. Items which are defective or have deteriorated are neither repaired nor replaced.

5. Shortages are not allowed.

6. At the start of the production cycle the system is in the in-control state, i.e., it does not produce any defective items.

7. The elapsed time before the process shifts to the out-of-control state is an exponentially distributed random variable with mean $1 / \mu$ (see figure 2 ).

8. During the out-of-control state, $\alpha$ of the total items produced will be defective.

9. Once a shift has occurred, the process will stay in the out-of-control state unless discovered (by inspection) and restored.

10. The scheduled maintenance inspections are performed at equal intervals.

11. The cost of restoration is a function of the duration of the out-of-control state, i.e., of $\tau$ (see figure 2).

12. Scheduled inspections and restorations are error-free and instantaneous.

\subsection{The inventory equations}

The methodology adopted in this paper involves a number of steps. First, the differential inventory equations for all the periods are developed. Next, these differential 


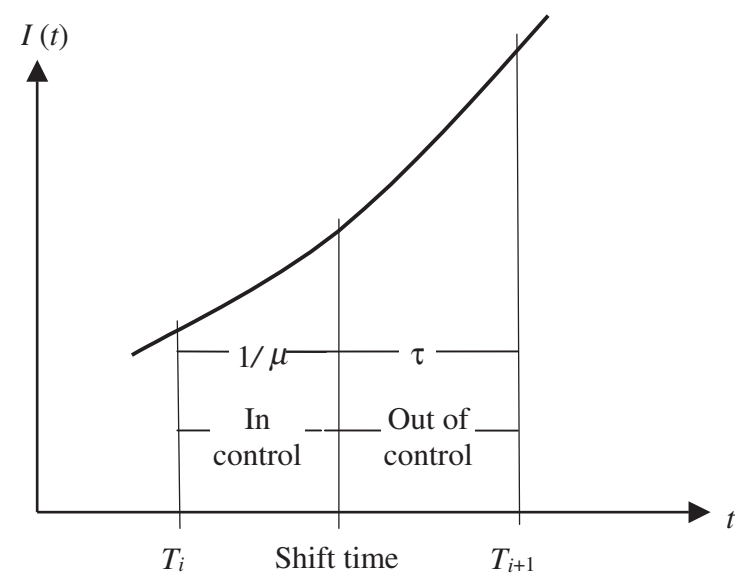

Figure 2. Process shift during a production cycle.

equations are solved to formulate the cost model. Finally, a heuristic numerical algorithm is proposed to search for the minimum-cost solution. The details of this methodology are discussed below.

In order to develop the differential inventory equations, we need to define the two stages of the production-inventory cycle shown in figure 1, a simplified representation of the production cycle. The effects of deterioration and the production of defective items are ignored in figure 1 (hence the approximate symbols for the slopes). The two stages are the production period $\left[0, t_{1}\right]$ and the consumption period $\left[t_{1}, t_{2}\right]$.

3.3.1. Production period $\left[0, t_{1}\right]$. During this stage, the inventory of good items increases due to production but decreases due to demand, deterioration, and the production of defective items. Thus, the inventory differential equation (IDE) is

$$
\frac{\mathrm{d} I}{\mathrm{~d} t}=P(t)-D(t)-\theta I(t)-E(N) .
$$

As shown in appendix A, the expected number of defective items produced per unit time is given by

$$
E(N)=\frac{a \alpha}{t_{1}}\left\{t_{1}+\frac{n}{\mu} \mathrm{e}^{-\mu\left(t_{1} / n\right)}-\frac{n}{\mu}\right\}+\frac{\alpha b A \mu}{t_{1}}\left\{\frac{t_{1}}{\lambda+\mu}-\frac{n}{(\lambda+\mu)^{2}}+\frac{n}{(\lambda+\mu)^{2}} \mathrm{e}^{-(\lambda+\mu)\left(t_{1} / n\right)}\right\} .
$$

In appendix B, we show that the inventory level during the production period is given by

$$
\begin{aligned}
I(t)= & \frac{a}{\theta}\left(1-\mathrm{e}^{-\theta t}\right)+\frac{A(b-1)}{\theta-\lambda}\left[\mathrm{e}^{-\lambda t}-\mathrm{e}^{-\theta t}\right] \\
- & {\left[\frac{a \alpha}{t_{1}}\left\{t_{1}+\frac{n}{\mu} \mathrm{e}^{-\mu\left(t_{1} / n\right)}-\frac{n}{\mu}\right\}+\frac{\alpha b A \mu}{t_{1}}\left\{\frac{t_{1}}{\lambda+\mu}-\frac{n}{(\lambda+\mu)^{2}}+\frac{n}{(\lambda+\mu)^{2}}\right.\right.} \\
& \left.\left.\times \mathrm{e}^{-(\lambda+\mu)\left(t_{1} / n\right)}\right\}\right] \frac{\left(1-\mathrm{e}^{-\theta t}\right)}{\theta}, \quad 0 \leq t \leq t_{1} .
\end{aligned}
$$


3.3.2. Consumption period $\left[t_{1}, t_{2}\right]$. The IDE during the consumption period, with no production and subsequently no reduction in the inventory level due to production of defective items, is given by

$$
\frac{\mathrm{d} I}{\mathrm{~d} t}=-D(t)-\theta I(t) .
$$

As shown in appendix $\mathrm{C}$, the inventory level during the consumption period is described by

$$
I(t)=\frac{A}{\lambda-\theta} \mathrm{e}^{-\lambda(t)}\left[1-\mathrm{e}^{-(\lambda-\theta)\left(t_{2}-t\right)}\right], \quad t_{1} \leq t \leq t_{2} .
$$

Since $I\left(t_{1}\right)=I_{\mathrm{m}}$, substituting $t=t_{1}$ into (3) gives

$$
\begin{aligned}
I_{\mathrm{m}}=\frac{a}{\theta}(1 & \left.-\mathrm{e}^{-\theta t_{1}}\right)+\frac{A(b-1)}{\theta-\lambda}\left[\mathrm{e}^{-\lambda t_{1}}-\mathrm{e}^{-\theta t_{1}}\right] \\
- & {\left[\frac{a \alpha}{t_{1}}\left\{t_{1}+\frac{n}{\mu} \mathrm{e}^{-\mu\left(t_{1} / n\right)}-\frac{n}{\mu}\right\}+\frac{\alpha b A \mu}{t_{1}}\right.} \\
& \left.\times\left\{\frac{t_{1}}{\lambda+\mu}-\frac{n}{(\lambda+\mu)^{2}}+\frac{n}{(\lambda+\mu)^{2}} \mathrm{e}^{-(\lambda+\mu)\left(t_{1} / n\right)}\right\}\right] \frac{\left(1-\mathrm{e}^{-\theta t_{1}}\right)}{\theta} .
\end{aligned}
$$

In line with $\mathrm{Su}$ and Lin (2001), a time shift of $t_{1}$ periods is applied to (4), corresponding to the consumption period's start time. Thus, substituting $t=0$ into (5) produces

$$
I_{\mathrm{m}}=\frac{A}{\lambda-\theta}\left[1-\mathrm{e}^{-(\lambda-\theta) t_{2}}\right]
$$

Equating (6) with (7), we obtain

$$
\begin{aligned}
& \frac{A}{\lambda-\theta}\left[1-\mathrm{e}^{-(\lambda-\theta) t_{2}}\right] \\
& =\frac{a}{\theta}\left(1-\mathrm{e}^{-\theta t_{1}}\right)+\frac{A(b-1)}{\theta-\lambda}\left[\mathrm{e}^{-\lambda t_{1}}-\mathrm{e}^{-\theta t_{1}}\right] \\
& -\left[\frac{a \alpha}{t_{1}}\left\{t_{1}+\frac{n}{\mu} \mathrm{e}^{-\mu\left(t_{1} / n\right)}-\frac{n}{\mu}\right\}+\frac{\alpha b A \mu}{t_{1}}\right. \\
& \left.\times\left\{\frac{t_{1}}{\lambda+\mu}-\frac{n}{(\lambda+\mu)^{2}}+\frac{n}{(\lambda+\mu)^{2}} \mathrm{e}^{-(\lambda+\mu)\left(t_{1} / n\right)}\right\}\right] \frac{\left(1-\mathrm{e}^{-\theta t_{1}}\right)}{\theta} .
\end{aligned}
$$

Denoting the right-hand side of (8) by $Q$, and solving for $t_{2}$, we derive the following expression for the length of the cycle time:

$$
t_{2}=\frac{1}{\theta-\lambda} \ln \left[\frac{A+(\theta-\lambda) Q}{A}\right] \text {. }
$$

\subsection{The cost components}

The setup cost per unit time, $C_{\mathrm{S}}$, is

$$
C_{\mathrm{S}}=\frac{k_{\mathrm{s}}}{t_{2}} .
$$


A total of $n$ inspections are carried out during the production stage $\left[0, t_{1}\right]$ of each cycle. For a fixed cost $k_{\mathrm{f}}$ per inspection, the total inspection cost per unit time, $C_{\mathrm{F}}$, is

$$
C_{\mathrm{F}}=\frac{n k_{\mathrm{f}}}{t_{2}}
$$

Deterioration cost, which is applicable to both stages of the production cycle, is

$$
C_{\mathrm{D}}=\frac{k_{\mathrm{d}}}{t_{2}} \int_{0}^{t_{2}} \theta I(t) \mathrm{d} t .
$$

Inventory holding cost is also applicable to both stages of the production cycle, as described by

$$
C_{\mathrm{H}}=\frac{k_{\mathrm{h}}}{t_{2}} \int_{0}^{t_{2}} I(t) \mathrm{d} t
$$

Quality cost is the cost incurred due to the production of defective items during the out-of-control phase of the process. This cost is applicable only to the first stage of the production cycle

$$
C_{\mathrm{Q}}=\frac{k_{\mathrm{q}} E(N) t_{1}}{t_{2}} .
$$

The restoration cost $C_{\mathrm{R}}$ is the cost incurred for maintenance performed, in order to restore the system from an out-of-control state back to an in-control state. This cost is given by Lee and Rosenblatt (1989) as

$$
C_{\mathrm{R}}=\frac{1}{t_{2}}\left\{\sum_{i=0}^{n-1} \int_{T_{i}}^{T_{i+1}} \mu R(\tau) \mathrm{e}^{-\mu\left(T_{i+1}-T_{i}-\tau\right)} \mathrm{d} \tau\right\} .
$$

Assuming equal inspection intervals, $T_{i+1}-T_{i}=t_{1} / n$, and based on the forgetfulness property of the exponential distribution, (15) can be written as

$$
C_{\mathrm{R}}=\frac{n}{t_{2}}\left[\int_{0}^{t_{1} / n} \mu R(\tau) \mathrm{e}^{-\mu\left[\left(t_{1} / n\right)-\tau\right]} \mathrm{d} \tau\right] .
$$

The total cost per unit time $T C$ is the sum of all the individual costs defined by (10)-(16)

$$
T C=C_{\mathrm{S}}+C_{\mathrm{F}}+C_{\mathrm{D}}+C_{\mathrm{H}}+C_{\mathrm{Q}}+C_{\mathrm{R}}
$$

It is evident that a closed-form optimum solution of the above equation is not practical. To minimize this function we employ a heuristic numerical solution algorithm using an integer search for the number of inspections $n$ and a line search for the corresponding production period $t_{1}$. In the following section, the algorithm is described, a numerical example is solved, and sensitivity analysis is performed. 


\section{Solution algorithm}

\subsection{Algorithm description}

In order to minimize $T C$, the algorithm goes through the following steps:

1. Substitute the given parameter numerical values into (10)-(16), and simplify them by numerically integrating all integrals and combining similar terms to get $T C$ from (17) as a function of $n, t_{1}$ and $t_{2}$.

2. Use (8) and (9) to find $t_{2}$ as a function of $t_{1}$.

3. Substitute $t_{2}$ back into (17) to get $T C=\mathrm{f}\left(n, t_{1}\right)$.

4. Set $n=1$.

5. Use the golden section search method to find $t_{1}\left(t_{1}^{*}\right)$ that minimizes $T C$, and subsequently $T C(n)$.

6. Use the equation obtained in step 2 to calculate $t_{2} *$.

7. Set $n=n+1$, and then repeat steps 5 and 6 to obtain $T C(n+1)$.

8. If $T C(n+1)<T C(n)$, go to step 7; otherwise, go to step 9 .

9. Stop. $T C(n)$ is the best heuristic total cost $T C^{*}$, and the corresponding $t_{1}(n)$ and $t_{2}(n)$ are the best production and cycle periods, i.e., $t_{1}{ }^{*}$ and $t_{2}{ }^{*}$.

The cost function (17) is nonlinear and involves both continuous and integer variables. The first three steps of the algorithm are designed to simplify the cost function as much as possible. However, the resulting function $T C$ is still extremely complicated. Thus, it is difficult to analytically test the convexity of the cost function. This fact and the use of numerical integration mean that the optimality of the solution cannot be guaranteed. Nonetheless, extensive numerical experiments indicate that $T C$ is piece-wise convex. Empirical experiments with several solved problems indicate that the algorithm is robust and produces high-quality solutions. After solving each problem by the algorithm, several other feasible solutions were randomly generated. In each of the tested problems, the solution obtained by the heuristic algorithm yielded the minimum total cost.

\subsection{A numerical example}

The following values for the parameters for a production-inventory system, adapted from Su and Lin (2001) and Lee and Rosenblatt (1989), will be used:

$$
\begin{array}{lll}
\alpha=500 \text { units/week, } & A=100, & b=0.1, \\
k_{\mathrm{d}}=3 / \text { unit }, & k_{\mathrm{f}}=4, & k_{\mathrm{h}}=1 / \text { unit/week, } \\
k_{\mathrm{q}}=5, & k_{s}=100 / \text { cycle }, & R(\tau)=10+0.15 \tau, \\
\alpha=0.2, & \lambda=0.3, & \mu=0.1, \\
\theta=0.05 . & &
\end{array}
$$

MATHEMATICA was used for the simplification of equations and a program was written in FORTRAN to perform the steps of the numerical algorithm. The results are summarized in table 1. From table 1, the best heuristic solution is obtained with one inspection and a total cost of $\$ 121.16$ per week.

Sensitivity analysis has been performed on the results of the example. One parameter was changed at a time (both decreased and increased) and the effect on the optimal solution was determined. Twenty-eight problems were solved, and sensitivity analysis results are summarized in table 2 . The model's sensitivity 
Table 1. Solution of the numerical example.

\begin{tabular}{llcc}
\hline$n$ & $T C$ & $t_{1}$ & $t_{2}$ \\
\hline 1 & $121.16^{*}$ & 0.4154 & 2.2123 \\
2 & 121.96 & 0.4283 & 2.3127 \\
\hline
\end{tabular}

Table 2. Sensitivity analysis for the given example.

\begin{tabular}{|c|c|c|c|c|c|c|}
\hline Parameter & Original value & New values & $n$ & $T C$ & $t_{1}$ & $t_{2}$ \\
\hline \multirow[t]{2}{*}{$a$} & 500 & 450 & 1 & 120.3 & 0.4755 & 2.2318 \\
\hline & & 550 & 1 & 121.95 & 0.3684 & 2.1950 \\
\hline \multirow[t]{2}{*}{$A$} & 100 & 90 & 1 & 114.35 & 0.3925 & 2.4296 \\
\hline & & 110 & 1 & 127.53 & 0.4403 & 2.0492 \\
\hline \multirow[t]{2}{*}{$b$} & 0.1 & 0.09 & 1 & 121.14 & 0.4164 & 2.2127 \\
\hline & & 0.11 & 1 & 121.17 & 0.4144 & 2.2119 \\
\hline \multirow[t]{2}{*}{$\theta$} & 0.05 & 0.045 & 1 & 119.99 & 0.4188 & 2.5527 \\
\hline & & 0.055 & 1 & 122.31 & 0.4122 & 2.172 \\
\hline \multirow{2}{*}{$\mu$} & 0.1 & 0.09 & 1 & 120.95 & 0.4175 & 2.2159 \\
\hline & & 0.11 & 1 & 121.37 & 0.4151 & 2.2086 \\
\hline \multirow[t]{2}{*}{$\lambda$} & 0.3 & 0.27 & 1 & 123.94 & 0.4031 & 2.0454 \\
\hline & & 0.33 & 1 & 118.06 & 0.4368 & 2.4815 \\
\hline \multirow[t]{2}{*}{$\alpha$} & 0.2 & 0.18 & 1 & 120.96 & 0.4158 & 2.2163 \\
\hline & & 0.22 & 1 & 121.35 & 0.4151 & 2.2083 \\
\hline \multirow[t]{2}{*}{$k_{\mathrm{d}}$} & 3 & 1 & 1 & 114.73 & 0.4428 & 2.4115 \\
\hline & & 5 & 1 & 127.27 & 0.3932 & 2.0575 \\
\hline \multirow[t]{2}{*}{$k_{\mathrm{h}}$} & 1 & 0.8 & 1 & 107.91 & 0.4787 & 2.6884 \\
\hline & & 1.2 & 1 & 133.12 & 0.3745 & 1.9317 \\
\hline \multirow[t]{2}{*}{$k_{\mathrm{q}}$} & 5 & 4 & 1 & 120.77 & 0.4166 & 2.2207 \\
\hline & & 6 & 1 & 121.55 & 0.41425 & 2.204 \\
\hline \multirow[t]{2}{*}{$k_{\mathrm{s}}$} & 100 & 80 & 1 & 111.26 & 0.3614 & 1.8464 \\
\hline & & 120 & 1 & 129.44 & 0.4741 & 2.6512 \\
\hline \multirow[t]{2}{*}{$k_{\mathrm{f}}$} & 4 & 0.1 & 6 & 118.02 & 0.4089 & 2.1783 \\
\hline & & 10 & 1 & 123.8 & 0.4322 & 2.333 \\
\hline \multirow[t]{4}{*}{$R(\tau)$} & $10+0.15 \tau$ & $1+0.15 \tau$ & 1 & 120.99 & 0.4152 & 2.2104 \\
\hline & & $5+0.15 \tau$ & 1 & 121.07 & 0.4153 & 2.2112 \\
\hline & & $10+0.1 \tau$ & 1 & 121.16 & 0.4154 & 2.2123 \\
\hline & & $10+0.2 \tau$ & 1 & 121.16 & 0.4154 & 2.2123 \\
\hline
\end{tabular}

to changes in input parameters is indicated by the range of $T C$ values in table 2 (107.91-133.12). Computation times in FORTRAN were negligible and practically indifferent for all 29 problems.

The total cost $T C$ was negatively correlated with $\lambda$ and positively correlated with all other parameters. The value of $T C$ was most sensitive to changes in $k_{\mathrm{h}}$, and least sensitive to changes in $b$. The production period $t_{1}$ was positively correlated with $A, \lambda, k_{\mathrm{s}}, k_{\mathrm{f}}$, and $R(\tau)$ and negatively correlated with all other parameters. The value of $t_{1}$ was most sensitive to $k_{\mathrm{s}}$, and least sensitive to $\alpha$ and $R(\tau)$. The cycle time $t_{2}$ was positively correlated with $\lambda, k_{\mathrm{s}}, k_{\mathrm{f}}$, and $R(\tau)$ and negatively correlated with all other parameters. The value of $t_{2}$ was most sensitive to $k_{\mathrm{s}}$ and $k_{\mathrm{h}}$, and least sensitive to $b$ and $R(\tau)$. Overall, the model is most sensitive to changes in $k_{\mathrm{s}}$ and $k_{\mathrm{h}}$, and least sensitive to changes in $b$ and $R(\tau)$. 


\section{Conclusions}

In this paper, we presented a model and a solution algorithm for incorporating quality and maintenance aspects into a production-inventory system for deteriorating items. Several possible extensions and improvements provide opportunities for future research. In general, closed-form solutions are rare in models that include the quality considerations. Because of the complexity of such models one normally has to resort to heuristic numerical solutions.

Further work could result in a model that is representative of many practical situations and that is useful as a decision making tool for management. Future research can be divided into two categories: extending the model, and improving the solution. Model extension possibilities include consideration of: inventorylevel-dependent production rate, shortages, non-instantaneous inspections/restorations, random demand, random deterioration rate, and random fraction of defects during the out-of-control phase. Possible solution improvement alternatives could be based on meta-heuristics such as genetic algorithms, tabu search, simulated annealing, or some other combination of optimization and numerical algorithms.

\section{Acknowledgments}

The authors are grateful to King Fahd University of Petroleum and Minerals for supporting this research effort. They also thank two anonymous referees for constructive comments and suggestions.

\section{Appendix A. Derivation of (2)}

Using assumptions 7 and 8, the expected number of defective items produced per unit time during the production interval $\left(0, t_{1}\right)$ is given by

$$
E(N)=\frac{1}{t_{1}} \sum_{i=0}^{n-1} \int_{T_{i}}^{T_{i+1} 1} \alpha P(t)\left(T_{i+1}-T_{i}-t\right) \mu \mathrm{e}^{-\mu t} \mathrm{~d} t .
$$

Substituting $P(t)=a+b D(t), D(t)=A \mathrm{e}^{-\lambda t}$, and $T_{i+1}-T_{i}=t_{1} / n$ leads to

$$
\begin{gathered}
E(N)=\frac{n}{t_{1}} \int_{0}^{t_{1} / n} \alpha\left(a+b A \mathrm{e}^{-\lambda t}\right)\left(\frac{t_{1}}{n}-t\right) \mu \mathrm{e}^{-\mu t} \mathrm{~d} t, \\
E(N)=\frac{n}{t_{1}}\left[\begin{array}{c}
\int_{0}^{t_{1} / n} \alpha a \mu \frac{t_{1}}{n} \mathrm{e}^{-\mu t} \mathrm{~d} t+\int_{0}^{t_{1} / n} \alpha b A \mu \frac{t_{1}}{n} \mathrm{e}^{-(\lambda+\mu) t} \mathrm{~d} t \\
-\int_{0}^{t_{1} / n} \alpha a \mu t \mathrm{e}^{-\mu t} \mathrm{~d} t-\int_{0}^{t_{1} / n} \alpha b A \mu t \mathrm{e}^{-(\lambda+\mu) t} \mathrm{~d} t
\end{array}\right] .
\end{gathered}
$$


Integrating, we obtain

$$
\begin{aligned}
E(N)= & \alpha a\left(1-\mathrm{e}^{-\mu t_{1} / n}\right)+\frac{\alpha b A \mu}{\lambda+\mu}\left(1-\mathrm{e}^{-(\lambda+\mu) t_{1} / n}\right)-\frac{n \alpha a \mu}{t_{1}}\left(-\frac{t_{1} \mathrm{e}^{-\mu t_{1} / n}}{n u}-\frac{\mathrm{e}^{-\mu t_{1} / n}}{u^{2}}+\frac{1}{u^{2}}\right) \\
& -\frac{n \alpha b A \mu}{t_{1}}\left(-\frac{t_{1} \mathrm{e}^{-(\lambda+\mu) t_{1} / n}}{n(\lambda+u)}-\frac{\mathrm{e}^{-(\lambda+\mu) t_{1} / n}}{(\lambda+u)^{2}}+\frac{1}{(\lambda+u)^{2}}\right) .
\end{aligned}
$$

Simplifying and combining similar terms in (A4), we obtain (2).

\section{Appendix B. Derivation of (3)}

Substituting $P(t)=a+b D(t)$ and $D(t)=A \mathrm{e}^{-\lambda t}$ into (1), we obtain

$$
\frac{\mathrm{d} I}{\mathrm{~d} t}+\theta I(t)=a+(b-1) A \mathrm{e}^{-\lambda t}-E(N)
$$

Multiplying both sides by $\mathrm{e}^{\theta t}$ produces

$$
\frac{\mathrm{d} I}{\mathrm{~d} t}\left[\mathrm{e}^{\theta t} I(t)\right]=a \mathrm{e}^{\theta t}+(b-1) A \mathrm{e}^{(\theta-\lambda) t}-E(N) \mathrm{e}^{\theta t} .
$$

Integrating both sides and dividing by $\mathrm{e}^{\theta t}$ leads to

$$
I(t)=\frac{a}{\theta}+\frac{(b-1) A}{\theta-\lambda} \mathrm{e}^{-\lambda t}-\frac{E(N)}{\theta}+C_{1} \mathrm{e}^{-\theta t} .
$$

Using the boundary condition $I(0)=0$, we obtain the value of the integration constant $C_{1}$

$$
C_{1}=-\frac{a}{\theta}-\frac{(b-1) A}{\theta-\lambda}+\frac{E(N)}{\theta} .
$$

Substituting $C_{1}$ from (B4) and $E(N)$ from (2) into (B3) and simplifying, we obtain (3).

\section{Appendix C. Derivation of (5)}

Substituting $D(t)=A \mathrm{e}^{-\lambda t}$, (4) can be written as

$$
\frac{\mathrm{d} I}{\mathrm{~d} t}=-A \mathrm{e}^{-\lambda t}-\theta I(t)
$$

Rearranging and multiplying both sides by $\mathrm{e}^{\theta t}$, we obtain

$$
\frac{\mathrm{d} I}{\mathrm{~d} t}\left[\mathrm{e}^{\theta t} I(t)\right]=-A \mathrm{e}^{(\theta-\lambda) t}
$$

Integrating both sides and dividing by $\mathrm{e}^{\theta}$, produces

$$
I(t)=\frac{A \mathrm{e}^{-\lambda t}}{\lambda-\theta}+C_{2} \mathrm{e}^{-\theta t} .
$$

Using the boundary condition $I\left(t_{2}\right)=0$, we obtain the following value of $C_{2}$

$$
C_{2}=-\frac{A \mathrm{e}^{(\theta-\lambda) t_{2}}}{\lambda-\theta} .
$$

Substituting $C_{2}$ from (C4) into (C3) and simplifying, we obtain (5). 


\section{References}

Abad, P.L., Optimal lot size for perishable goods under conditions of finite production and partial back ordering and lost sales. Comp. Ind. Eng., 2000, 38, 457-465.

Ben-Daya, M., Integrated production maintenance and quality model for imperfect processes. IIE Trans., 1999, 31, 491-501.

Benkherouf, L. and Mahmoud, M.G., On an inventory model for deteriorating items with increasing time-varying demand and shortages. J. the Op. Res. Soc., 1996, 47, $188-200$.

Chu, P. and Chen, P.S., A note on inventory replenishment policies for deteriorating items in an exponentially declining market. Com. and Oper. Res., 2002, 29, 1827-1842.

Goswami, A. and Chaudhuri, K.S., Variations of order-level inventory model for deteriorating items. Int. J. Prod. Econ., 1992, 27, 111-117.

Goyal, S.K. and Giri, B.C., Recent trends in modelling of deteriorating inventory. Euro. $J$. Oper. Res., 2001, 134, 1-16.

Hariga, M., Lot-sizing models for deteriorating items with time-dependent demand. Int. J. Sys. Sci., 1995, 26, 2391-2401.

Hwang, H.S., Inventory models for both deteriorating items and ameliorating items. Com. and Ind. Eng., 1999, 37, 257-260.

Iravani, S.M.R. and Duenyas, I., Integrated maintenance and production control of a deteriorating production system. IIE Trans., 2002, 34, 423-435.

Lee, H.L. and Rosenblatt, M.J.,A production and maintenance model with restoration cost dependent on detection delay. IIE Trans., 1989, 21, 368-375.

Misra, R., Optimal production lot size model for a system with deteriorating inventory. Int. $J$. Prod. Res., 1975, 13, 495-505.

Padmanabhan, G. and Vrat, P., EOQ models for perishable items under stock dependent selling rate. Euro. J. Op. Res., 1995, 86, 281-292.

Posner, M.J.M., Breg, M. and Zhiao, H., Production-inventory systems with unreliable machines. Op. Res., 1994, 42, 111-118.

Rahim, M.A., Joint determination of production quantity, inspection schedule, and control chart design. IIE Trans., 1994, 26, 2-11.

Rahim, M.A. and Ben-Daya, M., Joint determination of production quantity, inspection schedule, and quality control for an imperfect process with deteriorating products. J. Op. Res. Soc., 2001, 52, 1370-1378.

$\mathrm{Su}, \mathrm{C} .-\mathrm{T}$. and Lin, C.-W., A production inventory model which considers the dependence of production rate on demand and inventory level. Prod. Plan. Con., 2001, 12, 69-75.

Wang, C.-H. and Sheu, S.-H., Simultaneous determination of the optimal productioninventory and product inspection policies for a deteriorating production system. Com. and Op. Res., 2001, 28, 1093-1110.

Wang, S.-P., An inventory replenishment policy for deteriorating items with shortages and partial backlogging. Com. and Op. Res., 2002, 29, 2043-2051.

Wee, H.-M., Deterministic lot size inventory model for deteriorating items with shortages and a declining market. Com. and Op. Res., 1995, 22, 345-356. 